University of Nebraska - Lincoln

DigitalCommons@University of Nebraska - Lincoln

Evaluating the perspectives of those with severe physical impairments while learning $\mathrm{BCl}$ control of a commercial augmentative and alternative communication paradigm

Kevin Pitt

Jonathan S. Brumberg

Follow this and additional works at: https://digitalcommons.unl.edu/specedfacpub

Part of the Analytical, Diagnostic and Therapeutic Techniques and Equipment Commons, and the Special Education and Teaching Commons

This Article is brought to you for free and open access by the Department of Special Education and Communication Disorders at DigitalCommons@University of Nebraska - Lincoln. It has been accepted for inclusion in Special Education and Communication Disorders Faculty Publications by an authorized administrator of DigitalCommons@University of Nebraska - Lincoln. 


\title{
Evaluating the perspectives of those with severe physical impairments while learning BCI control of a commercial augmentative and alternative communication paradigm
}

\author{
Kevin M. Pitt, $\mathrm{PhD},{ }^{1}$ and Jonathan S. Brumberg, $\mathrm{PhD}^{2}$ \\ 1 Department of Special Education and Communication Disorders, \\ University of Nebraska-Lincoln, Lincoln, Nebraska, USA \\ 2 Department of Speech- Language-Hearing: Sciences \& Disorders, \\ University of Kansas, Lawrence, Kansas, USA \\ Correspondence - Kevin M. Pitt, PhD kevin.pitt@unl.edu Department of Special Education and \\ Communication Disorders, University of Nebraska-Lincoln, Lincoln, Nebraska 68583, USA. \\ ORCID \\ Kevin M. Pitt, PhD http://orcid.org/0000-0003-3165-4093 \\ Jonathan S. Brumberg, PhD http://orcid.org/0000-0001-5739-968X
}

\begin{abstract}
Augmentative and alternative communication (AAC) techniques can provide access to communication for individuals with severe physical impairments. Brain-computer interface (BCI) access techniques may serve alongside existing AAC access methods to provide communication device control. However, there is limited information available about how individual perspectives change with motor-based BCIAAC learning. Four individuals with ALS completed 12 BCI-AAC training sessions in which they made letter selections during an automatic row-column scanning pattern via a motor-based BCI-AAC. Recurring measures were taken before and after each BCI-AAC training session to evaluate changes associated with BCI-AAC performance, and included measures of fatigue, frustration, mental effort, physical effort, device satisfaction, and overall ease of device control. Levels of pre- to post-fatigue were low for use of the BCI-AAC system. However, participants indicated different
\end{abstract}

Published in Assistive Technology 2021

doi:10.1080/10400435.2021.1949405

Copyright (c) 2021 RESNA; published by Taylor \& Francis. Used by permission. Accepted 21 June 2021; published 9 July 2021 
perceptions of the term fatigue, with three participants discussing fatigue to be generally synonymous with physical effort, and one mental effort. Satisfaction with the BCI-AAC system was related to BCI-AAC performance for two participants, and levels of frustration for two participants. Considering a range of person-centered measures in future clinical BCI-AAC applications is important for optimizing and standardizing BCI-AAC assessment procedures.

Keywords: brain-computer interface, augmentative and alternative communication, perspectives, fatigue, amyotrophic lateral sclerosis, satisfaction

Augmentative and alternative communication (AAC) devices seek to provide access to communication for a variety of individuals with heterogeneous cognitive-sensory-motor profiles, including those with severe physical impairments due to diagnoses such as cerebral palsy and amyotrophic lateral sclerosis (ALS). Communication access can be achieved through a combination of both high (e.g., eye gaze access to electronic device) and low technology (i.e., techniques not requiring an electronic device) access methods (Beukelman \& Light, 2020). Due to the large variety and level of cognitive-sensory motor strengths presented by individuals with severe physical impairments who may use AAC, there are some for whom current AAC access methods do not adequately meet their complex communication needs. It is therefore important that research on the development of new AAC access methods is conducted to bridge this gap and help ensure an efficient and effective form of communication for all (Fager et al., 2019).

A new high technology-based AAC access for those with severe physical impairments focuses on translating brain signals into communication device control. Noninvasive brain-computer interface access methods for AAC (BCI-AAC) are currently in development and have the potential to be viewed alongside existing AAC access methods as an option for communication device control by those with severe physical impairments (Brumberg et al., 2018). Noninvasive BCI-AAC techniques commonly employ electroencephalography (EEG), which records brain activity observable from the scalp using surface electrodes. Similar to existing AAC access techniques, a range of BCI-AAC methods are in development to provide communication device control, which can broadly be categorized into those targeting brain signals associated with sensory, or motor tasks. For instance, motor-based BCI-AAC techniques translate changes in brain activity associated with imagined 
or attempted target movements into communication device selections (e.g., Vaughan et al., 2006). In more detail, when the brain is at rest, neurons produce rhythmic and synchronized activity between 8 and $12 \mathrm{~Hz}$ known as the alpha rhythm (Pfurtscheller \& Da Silva, 1999; Pfurtscheller \& Neuper, 2009), which is commonly termed the murhythm when measured over sensorimotor areas of the brain (Kuhlman, 1978). An important property of the mu-rhythm is its change in power when the brain engages in processing information or performing physical or imagined motor tasks. Specifically, as neural synchronization decreases with attempted or imagined movements, then so does the overall power in the mu frequency band (at rest, synchronization and mu power increase; Pfurtscheller \& Da Silva, 1999). When decreases in neural synchrony are due to an event (e.g., cued performance of a motor task), it is known as event related desynchronization (Pfurtscheller \& Da Silva, 1999), which can be traced back to a specific event for translation into a computer command (e.g., select an item).

As research continues to advance in laboratory settings, recent efforts show an increasing focus on incorporating the perspectives of individuals who may use BCI-AAC in design and implementation (e.g., Blain-Moraes et al., 2012; Geronimo et al., 2015; Huggins et al., 2011; Pitt \& Brumberg, 2018a; Peters et al., 2016; Pitt \& Brumberg, 2020). An ineffective user-to-device match can increase the likelihood of device abandonment (Johnson et al., 2006). Therefore, similar to usercentered design which focuses on the individual, and how designs can meet the needs and requirements of target end users (Chavarriaga et al., 2017; Kübler et al., 2014), in the clinical realm, feature matching procedures for AAC consider an individual's strengths, preferences, barriers, and environment in relation to AAC access to help ensure an individual is matched to the method most likely to support communication success (Beukelman \& Light, 2020). For instance, AAC abonnement is more likely if an individual is matched to an AAC system they find difficult to use or does not match their cognitive abilities (Johnson et al., 2006).

Both user-centered design and feature matching considerations for BCI-AAC may be elucidated by understanding a user's experience (also known as "UX") during BCI-AAC use. For instance, while measures of speed and accuracy are still important for BCI-AAC development, other factors related to the user experience (e.g., levels of effort, frustration, 
and satisfaction) may highlight important factors not clearly indicated by more traditional performance measures. When considered in a feature-matching framework, the field of $\mathrm{AAC}$ and $\mathrm{BCI}$ can benefit from understanding the experiences of individuals using BCI-AAC to help identify the best match between an individual and a communication device (Bircanin et al., 2019). For example, after a period of training with different BCI-AAC systems that match the individual's profile the individual may wish to choose the system that potentially has lower accuracy, but is associated with lower workloads, possibly raising levels of personal satisfaction (e.g., Peters et al., 2016). Furthermore, evaluating the user experience may provide new direction for BCI-AAC development by identifying important factors for overall BCI-AAC acceptance, such as decreasing levels of frustration (Lorenz et al., 2014). To date, multiple tools for evaluating the user experience have been developed, with user experience research being a common feature in the field of human-computer interaction (see Kögel et al., 2019 for review). However, there is limited evaluation of end-user experience in the field of BCI-AAC, with existing research primarily focusing on factors related to usability (e.g., workload and satisfaction) versus appeal (e.g., factors related to motivation and frustration; Lorenz et al., 2014). Further, the majority of existing tools are not specifically designed to support the completion of individuals with severe physical impairments in relation to their experience with BCI-AAC (Andresen et al., 2016; Peters et al., 2016). Therefore, further work is needed to understand how individuals experience the use of BCI-AAC systems.

Current user-centered research suggests individuals may find BCIAAC use effortful and fatiguing (Fager et al., 2019) possibly increasing the risk for BCI-AAC abandonment. However, the vast majority of research that has obtained fatigue and effort feedback from individuals during $\mathrm{BCI}-\mathrm{AAC}$ trainings focused on just a single paradigm, the P30o-based BCI-AAC (e.g., Blain-Moraes et al., 2012; Peters et al., 2016). Therefore, as BCI-AAC devices include more than just P30obased approaches, procedures must account for how one's perception of BCI-AAC use may change not only during P3oo-based BCI-AAC training sessions but for other BCI-AAC techniques. Considering an individual's experiences with a range of BCI-AAC techniques is important since not everyone may be best suited to the same type of approach (Pitt \& Brumberg, 2018a), BCI-AAC (and AAC in general) are 
not a one-size-fits-all intervention, and ratings of factors such as mental effort may change between BCI-AAC techniques (Combaz et al., 2013; Geronimo et al., 2015). Motor based-BCI-AAC paradigms may especially benefit from understanding user perspectives given their increased training times in comparison to P300-based BCI-AAC methods which are necessary for supporting motor learning (e.g., Mak \& Wolpaw, 2009).

Motor-based BCI-AAC learning is influenced by motivational factors for individuals without neurological impairments (Friedrich et al., 2013) as well as those with ALS (Nijboer et al., 2010), with initial BCIAAC performance impacting an individual's level of interest in learning BCI-AAC control (Geronimo et al., 2015). Furthermore, individuals may report high levels of exhaustion during early BCI-AAC training (Friedrich et al., 2013), and some reports by individuals with ALS indicate frustration and dissatisfaction with motor-based BCI-AAC control during the early stages of motor learning (Nijboer et al., 2010), which may negatively impact communication outcomes (Johnson et al., 2006). Similar to motor learning of physical actions, it is likely that an individual's perception of motor-based BCI-AAC use may become more positive over time, as the individual learns BCI-AAC control (Geronimo et al., 2015). Therefore, elucidating an individual's evolving perspectives during BCI-AAC training may help inform clinical guidelines regarding how to best match an individual to an BCI-AAC device that is a good match in supporting their functional participation without being perceived as too difficult to use, especially during early intervention periods. Furthermore, while measures of satisfaction are an important component in person-centered assessment and intervention frameworks (Kübler et al., 2014; Peters et al., 2016), how a full range of factors such as fatigue, effort, frustration, and performance impact an individual's satisfaction with BCI-AAC use is currently unknown. Therefore, important considerations for BCI-AAC design, and potential strategies for tailoring intervention to maximize learning and minimizing the potential for abandonment are unclear. Thus, in the present study, we specifically examined how an individual's unique perspective regarding fatigue, frustration, mental effort, physical effort, overall effort and device satisfaction changed across $12 \mathrm{BCI}-\mathrm{AAC}$ training sessions in relation to BCI-AAC performance. We expected to find decreasing ratings of fatigue, frustration, and effort, 
and improved ratings of satisfaction with increasing BCI-AAC performance. We further expected decreasing levels of fatigue, frustration, and effort to be associated with increased levels of satisfaction.

\section{Methods}

The institutional review board at the University of Kansas approved this study. All participants providing informed consent and were financially compensated. The BCI-AAC task used as the framework for evaluating fatigue, frustration, mental effort, physical effort, and device satisfaction is described in detail in our prior work (Pitt \& Brumberg, 2021). However, while Pitt and Brumberg (2021) discussed the importance of initial assessment measures such as motor skills, cognition, fatigue, and motivation for predicting BCI-AAC performance, here we describe how recurring measures that describe how the participants experience with BCI-AAC changed during their 12-session training period. A summary of details from Pitt and Brumberg (2021) regarding BCI-AAC training, and performance, important for understanding the full context of this investigation are given below.

\section{The motor-based BCI-AAC system}

During this investigation, participants made letter selections during an automatic row-column scanning pattern from a $7 \times 5$ keyboard display, which included letters A-Z, space, and backspace, and participants were instructed to continue attempting to select the correct letter without using backspace for spelling errors. All BCI software was implemented in Python with the display specifically implemented using the Kivy framework (kivy.org). Calibrated decoding model weights were estimated using MATLAB, which were then stored and loaded into Python implementations of the same model for real-time decoding. Event-related desynchronization occurred when participants imagined or attempted a limb movement to signify an intent to select some screen item currently highlighted in the visual display during an automatic item scanning paradigm. The BCI-AAC then translated the desynchronization into an actual selection command for interpretation by the BCI-AAC system (Pitt \& Brumberg, 2021). EEG recording 
was collected using 62 active electrodes (g.HIAmp, g.tec) arranged according to the 10-10 standard (Oostenveld \& Praamstra, 2001), with a forehead ground and earlobe reference at a sampling rate of $256 \mathrm{~Hz}$. The BCI-AAC decoding framework used a regularized linear discriminant analysis algorithm with sensorimotor common spatial patterns as decoding features (Lotte \& Guan, 2010). The sensorimotor band was tailored to each participant per session based on visual inspection of the EEG power spectrum. The regularized common spatial patterns and linear discriminant analysis decoder weights were optimized offline based on calibration data and then stored for real-time application during active BCI-AAC tasks. Calibration data was obtained at the start of each session in order to train the decoder using 30 calibration trials for left-limb movement, right-limb movement and rest and all data were aligned to a photodiode trigger signal at the start of each trial. The final decoding model was based on either right versus rest or left versus rest, according to highest area under the curve and participant preference.

\section{$B C I-A A C$ training procedures}

During real-time (online) BCI-AAC control, participants copy spelled words by making letter selections during an automatic row-column scanning pattern using the calibrated BCI-AAC decoder. In this clinically based AAC paradigm, a selection box sequentially highlights each possible row while the individual is at rest with row selection occurring when the BCI-AAC detects event-related desynchronization associated with a motor action. Items were highlighted for a 2 second duration with an interstimulus duration of 1 second. Following row selection, each letter within the selected row is sequentially highlighted until the individual again performs the target motor action to select the highlighted letter. Visual feedback was provided to participants in the form of a circle/ellipse, which was overlaid on the current item. The circle became smaller in size as the BCI-AAC algorithm became more confident in predicting a "select" command and became bigger as confidence decreased. Participants 1, 2 and 4 were all able to sit normally in either a chair in the laboratory sound booth recording area, or at a chair in their home. In these cases, the computer monitor was positioned appropriately for desktop use. Participant 3 completed 
the study in a recliner in her home. In this case, we used a combination of recliner elevation and positioning of a rolling desk, with height and angle adjustments, to ensure the screen was visible. We additionally used a neck support to provide some relief for electrodes placed on the back of the head.

Participants completed 12 BCI-AAC training sessions, with each including approximately 300 real-time control trials which included all row/column selection opportunities, including classification of selection versus rest. Due to the additional time required for EEG and BCI-AAC set-up procedures and calibration, real-time data collection lasted approximately 20 minutes to allow time for completion of all study procedures and provide consistency between training sessions regarding duration of online BCI-AAC use. Overall session duration was not limited. Due to difficulties with traveling, participants A3 and A4 completed BCI-AAC training sessions in a quiet room within their home setting. In contrast, A1 and A2 completed training sessions in the laboratory (i.e., an electrically shielded booth, with the door open to allow for communication with the participant throughout the BCIAAC session). Participants A1, A2, and A4 completed training sessions approximately twice per week, with $\mathrm{A} 3$ completing training once per week. All participants were able to perform a motor movement for BCI-AAC control, with $\mathrm{P}_{1}, \mathrm{P}_{2}$, and $\mathrm{P} 3$ using an upper limb movement, and $\mathrm{P} 4$ a lower limb movement, see Pitt and Brumberg (2021) for further details.

\section{Participants}

Four individuals with a diagnosis of ALS (participants A1-A4, ages 3864, mean 52 years, two females, all right-handed) completed recurring number scale measures evaluating their perspectives of fatigue, satisfaction, mental effort and physical effort during BCI-AAC training. A summary of participant information is provided in Table 1, with scores from the ALS Cognitive Behavioral Screen (ALS-CBS; Woolley et al., 2010), the cognitive and motor portions of the BCI-AAC screener from Pitt and Brumberg (2020), and the ALS-Functional Rating Scale (ALS-FRS; Cedarbaum \& Stambler, 1997) completed by each participant before the first session. Participants did not demonstrate or report any vision or hearing impairment that may impair BCI-AAC use 
Table 1. Participant information.

\begin{tabular}{llllllll}
$\begin{array}{l}\text { Participant } \\
\text { Number }\end{array}$ & Diagnosis & Sex & $\begin{array}{l}\text { Age } \\
\text { (years) }\end{array}$ & $\begin{array}{l}\text { ALS- } \\
\text { CBS }\end{array}$ & $\begin{array}{l}\text { ALS- } \\
\text { FRS }\end{array}$ & $\begin{array}{l}\text { BCl screener: } \\
\text { Cognitive }\end{array}$ & $\begin{array}{l}\text { BCl screener: } \\
\text { Motor }\end{array}$ \\
\hline A1 & Bulbar ALS & F & 64 & 15 & 33 & 22 & No motor impairment. \\
A2 & Spinal ALS & M & 38 & 19 & 34 & 22 & Limited range of motion. Ambulatory with assistance. \\
A3 & Spinal ALS & F & 48 & 19 & 15 & 24 & Non-ambulatory, minimal movement of legs and thighs. \\
A4 & Spinal ALS & M & 57 & 14 & 26 & 24 & Ambulatory without assistance, limited fine motor control.
\end{tabular}

Information for individuals with ALS including total cognitive scores from 1) the ALS-CBS (maximum score of 20, with scores below 17 being indicative of concern for cognitive impairment; (Woolley, 2014), 2) the ALS-FRS (maximum score of 40 with lower scores indicating increased motor impairment), and 3) total cognitive scores (maximum score of 24) and descriptive motor results from the BCI-AAC screener.

and were without oculomotor impairment. All participants were able to respond verbally to question prompts and used speech as their primary communication method. However, A3 used an eye-gaze AAC device to support access social media and communication as needed. Further, participant A4 had recently purchased a knee switch for AAC access but had not yet begun training. Participants A1 and A2 did not use AAC technology.

\section{Recurring number scale measures of participant perspectives and satisfaction}

The participant feedback questionnaire (available in supplementary material A) was adapted from Peters et al. (2016). Specifically, a question on fatigue was added since individuals may experience fatigue during BCI-AAC use (Fager et al., 2019). Further, the number of questions was reduced to facilitate the collection of repeated measures. Finally, our feedback questionnaire utilized a 9-point number scale. Nine-point number scales are commonly used in assessing user experiences (Peters et al., 2016). However, while future research is needed, the 7-point number scale used by Peters et al. (2016) may have reduced effort in completion. During administration, verbal, and visual support (i.e., a printed version of the questionnaire that was readable by the participant) was provided. Further, during completion the examiner requested confirmation that recorded answers were accurate.

The feedback questionnaire was taken directly prior to (for fatigue only) and following (for all measures) each BCI-AAC training session 
to track changes associated with BCI-AAC learning. The questionnaire included measures of:

(1) Fatigue: A 9-point scale to ascertain the level of fatigue associated with BCI-AAC control before and after each session with 1 indicating "normal fatigue," through 9 indicating "extremely fatigued." Pre- to post-fatigue use was calculated by subtracting post-session fatigue ratings from pre-session fatigue ratings. Participants were also asked how they define the term fatigue to provide further insight into participant responses. All participants were able to complete this task verbally.

(2) Device Satisfaction: BCI-AAC device satisfaction was evaluated via a 9-point number scale with 1 indicating "very unsatisfied," through 9 "very satisfied."

(3) Frustration: Frustration controlling the BCI-AAC was evaluated via 9-point number scale with 1 indicating "very low," through 9 "very high".

(4) Physical and mental effort: Physical and mental effort associated with the BCI-AAC control were evaluated via 9-point number scale with 1 indicating "very low," through 9 "very high."

(5) Overall levels of effort: Overall level of effort (i.e., "how hard" they had to work), was evaluated via 9-point number scale of 1 indicating "very easy," through 9 "very hard."

Based upon the procedures of Nijboer et al. (2010), we used a Spearman's rank order correlation to identify the unique perspectives of our participants during BCI-AAC training. The Spearman's rank order correlation was calculated within each participant to evaluate the relationship between each participant's number scale measures and BCIAAC task performance for each of their 12 training sessions.

\section{BCI-AAC performance}

BCI-AAC performance was calculated via Cohen's Kappa using successes and failures for each selection opportunity compared to user intention (e.g., making a selection when needed, refraining from a selection when appropriate). Cohen's Kappa represents both the true positive rate (i.e., the BCI-AAC made a selection when the individual 
intended for the BCI-AAC to make a selection) and true negative rates (i.e., the BCI-AAC allowed the display to continue scanning through the items, and no selections were made while the participant was at rest), weighted by both true rates (positive and negative) and the false- positive rate (i.e., the BCI-AAC made a selection when the individual did not intend for the BCI-AAC to make a selection), and falsenegative rates (i.e., the $\mathrm{BCI}-\mathrm{AAC}$ did not make a selection when the individual tried to activate the system). A Cohen's Kappa value of o to 0.20 indicates no to slight agreement between the BCI-AAC output and user intention, 0.21 to 0.4 as fair agreement, 0.41 to 0.6 as moderate agreement, 0.61 to 0.8 as substantial agreement and .81 to 1 as almost perfect agreement (e.g., McHugh, 2012). In the current scanning paradigm participants are required to wait for items to be automatically highlighted before making infrequent selections, which skews traditional measures (e.g., percent classification accuracy) toward nonselection tasks. Therefore, Cohen's Kappa was utilized for tracking BCI-AAC learning as the metric reflects both true negative and true positive rates (Pitt \& Brumberg, 2021).

\section{Results}

Pertinent data regarding BCI-AAC performance results and learning trajectories are provided in Table 2 and Figures 1-4. Detailed BCIAAC performance results are presented in Pitt and Brumberg (2021). In summary, participants A1 (Kappa = 0.333; fair agreement) and A4 (Kappa = .199; no to slight agreement) achieved higher levels of BCIAAC performance in comparison to A2 (Kappa = 0.139; no to slight agreement) and A3 (Kappa = 0.01; below chance levels). The learning trajectory of A4 was associated with the largest slope (0.0347).

\section{Recurring measures}

Individual ratings for each session provided in supplemental material B. For clarity, the section below identifies only significant results, with summary statistics and significance for others measures in relation to BCI-AAC performance and satisfaction provided in Table 3. As 
Table 2. $\mathrm{BCl}-\mathrm{AAC}$ performance results for participants 1 to 4 .

\begin{tabular}{|c|c|c|c|c|c|}
\hline Participant & Mean & $S D$ & Range & $\begin{array}{l}\text { Learning } \\
\text { slope }\end{array}$ & $\begin{array}{l}\text { Description of performance } \\
\text { and notable } 95 \% \text { confidence intervals }\end{array}$ \\
\hline 1 & $\begin{array}{l}0.333 \\
\text { (fair } \\
\text { agreement) }\end{array}$ & 0.151 & $\begin{array}{l}0.020-0.544 \\
\text { (no to slight- } \\
\text { moderate } \\
\text { agreement) }\end{array}$ & 0.0023 & $\begin{array}{l}\text { She had a relatively flat learning trajectory after } \\
\text { rapidly increasing } \mathrm{BCI}-\mathrm{AAC} \text { performance } \\
\text { between sessions } 1 \text { and } 2 \text {. Her } 95 \% \text { confidence } \\
\text { interval range extended into the range of } \\
\text { substantial agreement for sessions } 6(0.641) \\
\text { and } 7 \text { ( } 0.651)\end{array}$ \\
\hline 2 & $\begin{array}{l}0.139 \\
\text { (no to slight } \\
\text { agreement) }\end{array}$ & .117 & $\begin{array}{l}-.051-0.340 \\
\text { (below chance- } \\
\text { moderate } \\
\text { agreement) }\end{array}$ & 0.0033 & $\begin{array}{l}\text { Overall, his performance was very variable. } \\
\text { His } 95 \% \text { confidence interval range extended } \\
\text { into the range of moderate agreement } \\
\text { for sessions } 1(0.438) \text { and } 4(0.551) .\end{array}$ \\
\hline 3 & $\begin{array}{l}-0.01 \\
\text { (below } \\
\text { chance } \\
\text { levels) }\end{array}$ & .096 & $\begin{array}{l}-.017-0.13 \\
\text { (below chance } \\
\text {-no to slight } \\
\text { agreement) }\end{array}$ & 0.0155 & $\begin{array}{l}\text { Although her performance varied, her learning } \\
\text { trajectory began at session } 3 \text {. A correlation } \\
\text { approached but did not reach significance } \\
\text { between session number and performance } \\
\left(r_{s}(10)=.517, p=.085\right) \text {. }\end{array}$ \\
\hline 4 & $\begin{array}{l}.199 \\
\text { (no to slight } \\
\text { agreement) }\end{array}$ & .177 & $\begin{array}{l}-0.05-0.47 \\
\text { (below chance } \\
\text {-no to } \\
\text { moderate } \\
\text { agreement) }\end{array}$ & 0.0347 & $\begin{array}{l}\text { His learning trajectory began at session } 3-4 \text {, } \\
\text { session number positively correlating to BCI-AAC } \\
\text { performance }\left(r_{s}(10)=.699, p<.05\right) \text {. Upper levels } \\
\text { with of moderate agreement were recorded for } \\
\text { the } 95 \% \text { confidence intervals of sessions } 10 \text { (.511) } \\
\text { and } 11(.591) \text {. He had the largest slope } \\
\text { associated with his BCI-AAC learning trajectory. }\end{array}$ \\
\hline
\end{tabular}

BCl-AAC performance results for Participants 1 to 4, indicating Kappa mean, standard deviation (SD), range, and slope of their participants learning trajectory over 12 sessions, along with a brief performance description. Further information can be found in Pitt and Brumberg (2021).

each participant demonstrated a unique learning trajectory (Table 2), the following results parallel the procedures of Nijboer et al. (2010) using within-subject analysis to elucidate specific user experiences.

\section{Recurring measures in relation to BCI-AAC performance}

Correlations between BCI-AAC accuracy and satisfaction were statistically significant and positive for participant $\mathrm{A} 1\left(\mathrm{r}_{\mathrm{s}}(10)=.651, p\right.$ $<.05$; Figure 1$)$ and A3 $\left(r_{s}(10)=.715, p<.05\right.$; Figure 2$)$.

\section{Recurring measures in relation to satisfaction}

Correlations between BCI-AAC satisfaction ratings and frustration with device control were statistically significant and negative for A2 $\left(r_{s}(10)=-.841, p<.05\right.$; Figure 3$)$, and A4 $\left(r_{s}(10)=-.702, p<.05\right.$; Figure 4). 


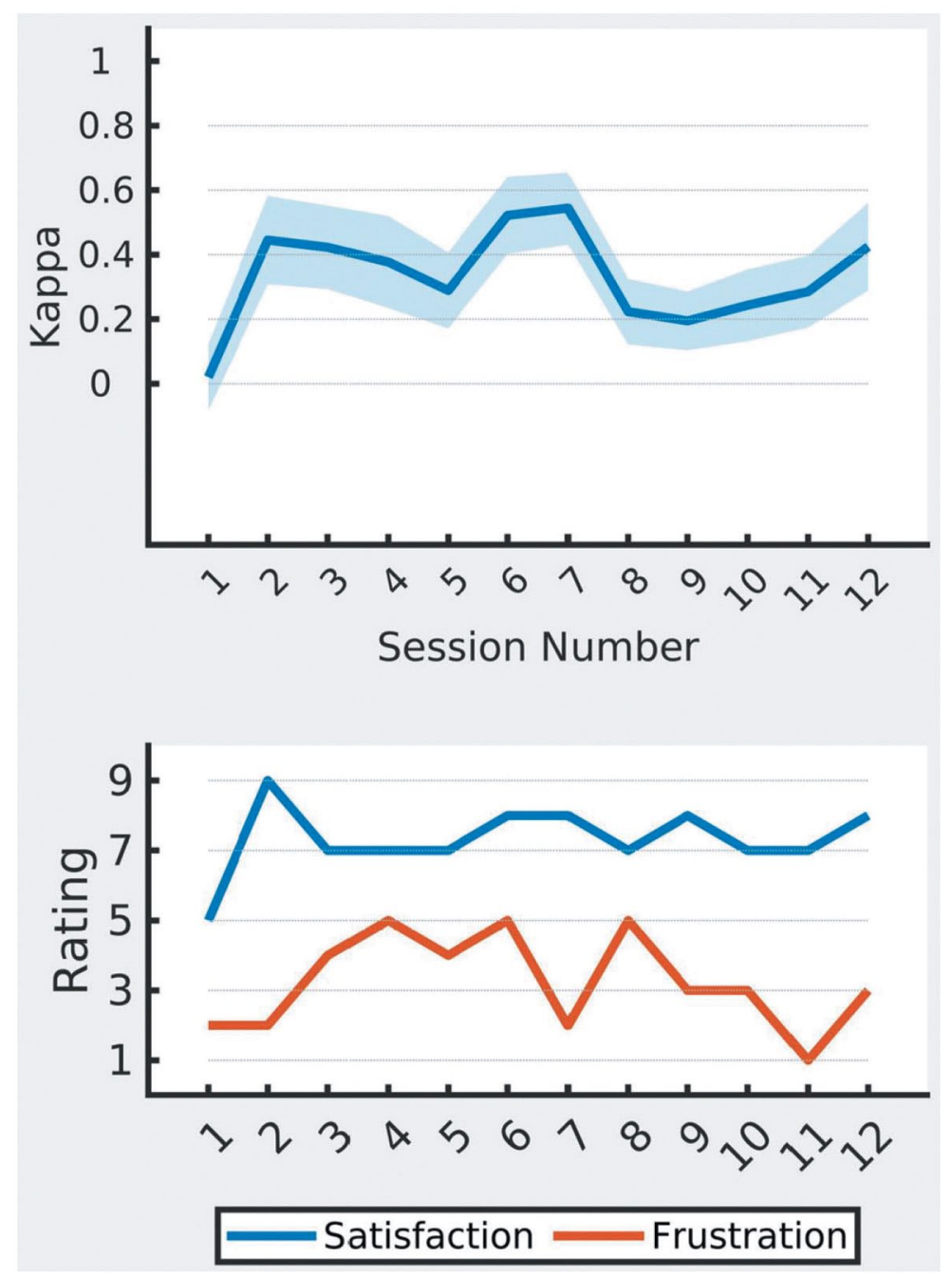

Figure 1. $\mathrm{BCl}-\mathrm{AAC}$ performance for $\mathrm{A} 1$, with $95 \%$ confidence intervals shown in the shaded blue area (top), along with number scale ratings of satisfaction and frustration with device control (bottom). A significant correlation was identified between $\mathrm{BCl}-\mathrm{AAC}$ accuracy and satisfaction.

\section{Definitions of fatigue}

Participants A1, A2, and A4 reported that they defined the term fatigue and physical effort generously synonymously. For instance, A4 reported he would define something as highly fatiguing if he ached the next day (e.g., after mowing the grass). However, in contrast, A3 reported that she defined the term fatigue and mental effort generously synonymously (e.g., fatigue after taking an exam). 


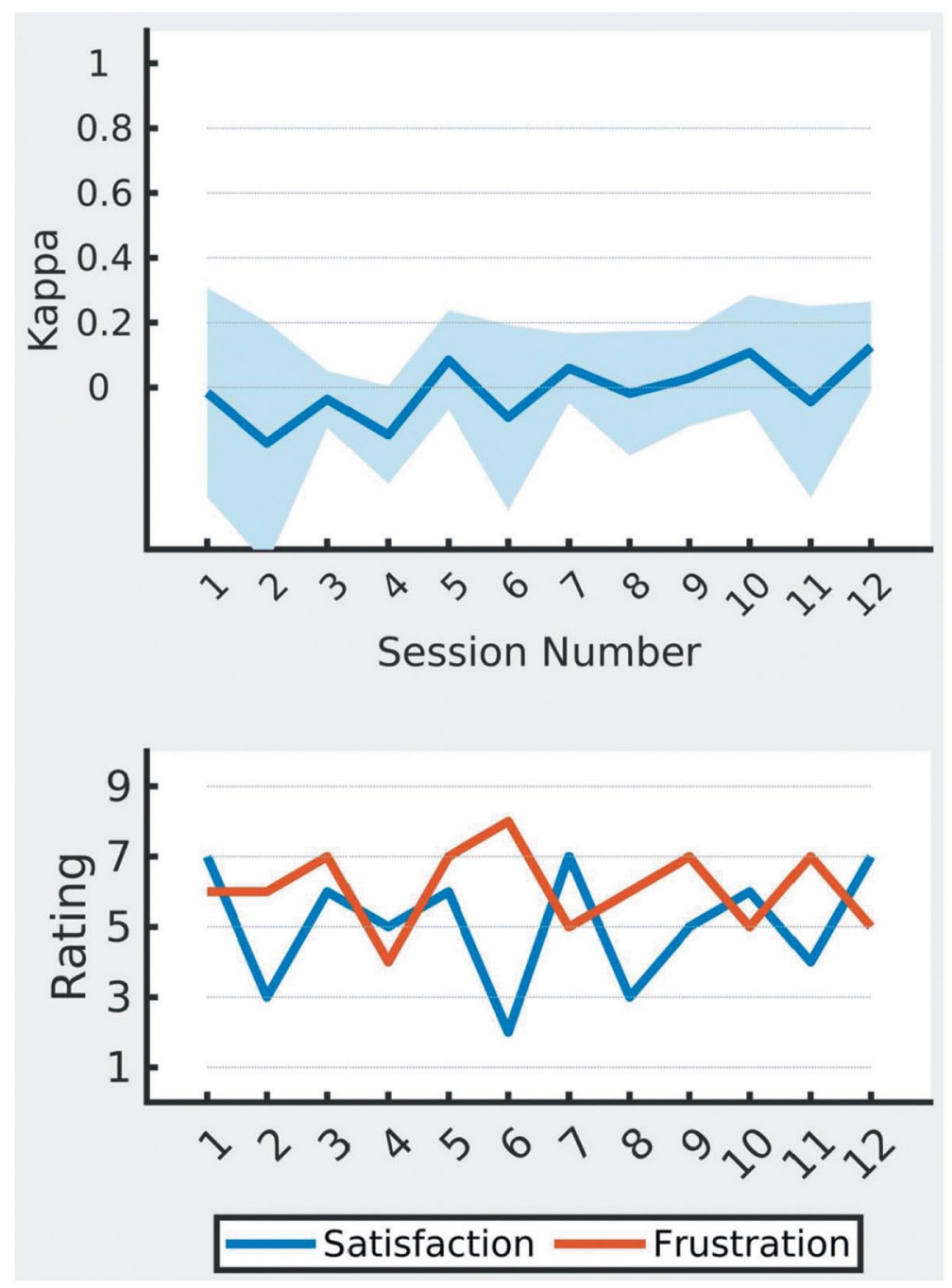

Figure 2. $\mathrm{BCl}-\mathrm{AAC}$ performance for $\mathrm{A} 3$, with $95 \%$ confidence intervals shown in the shaded blue area (top), along with number scale ratings of satisfaction and frustration with device control (bottom). A significant correlation was identified between $\mathrm{BCl}-\mathrm{AAC}$ accuracy and satisfaction.

\section{Discussion}

Traditionally BCI-AAC intervention studies have largely focused on accuracy-based performance outcomes (Pitt et al., 2019). While these $\mathrm{BCI}-\mathrm{AAC}$ paradigms and outcome measures are important for BCI-AAC development and laying the foundation for BCI-AAC development and the transition of BCI-AAC into clinical practice, it is important to consider person-centered, intrinsic (as well as extrinsic) factors that may 


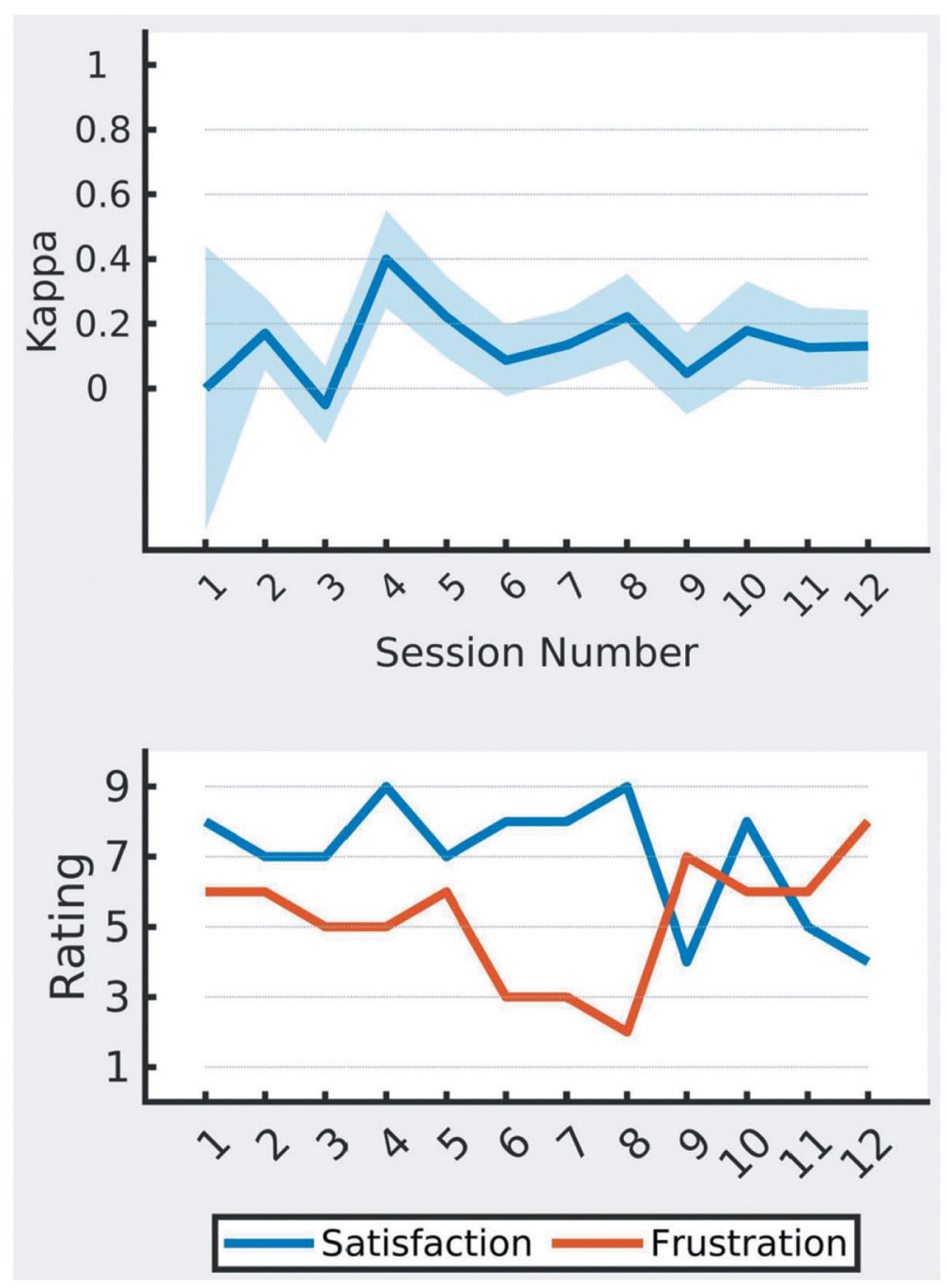

Figure 3. $\mathrm{BCl}-\mathrm{AAC}$ performance for $\mathrm{A} 2$, with $95 \%$ confidence intervals shown in the shaded blue area (top), along with number scale ratings of satisfaction and frustration with device control (bottom). A significant correlation was identified between $\mathrm{BCl}-\mathrm{AAC}$ frustration and satisfaction.

affect BCI-AAC outcomes in ways not easily quantified by traditional measures. In our study, we build upon past work supporting incorporation of participant feedback on BCI-AAC paradigms and designs and focus specifically on intrinsic, person-centered factors such as satisfaction, levels of frustration and multiple measures of effort (e.g., mental and physical effort), and explore individuals' definition of fatigue to aid interpretation of accuracy-based outcomes to identify factors influencing BCI-AAC performance and satisfaction. 


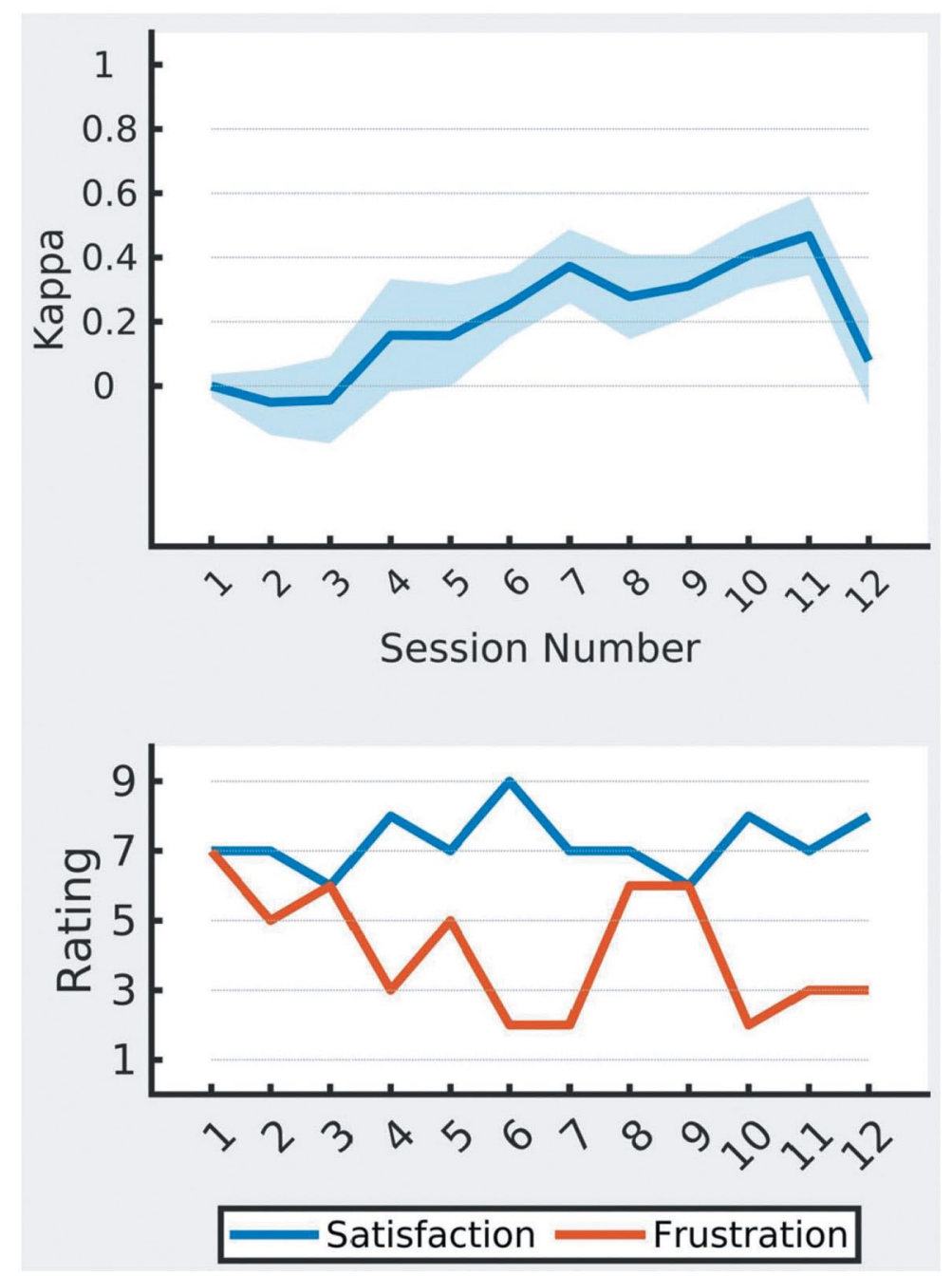

Figure 4. $\mathrm{BCl}-\mathrm{AAC}$ performance for $\mathrm{A} 4$, with $95 \%$ confidence intervals shown in the shaded blue area (top), along with number scale ratings of satisfaction and frustration with device control (bottom). A significant correlation was identified between $\mathrm{BCl}-\mathrm{AAC}$ frustration and satisfaction.

\section{Relationships Between Recurring Measures and BCI-AAC Performance}

The range of number scale ratings provided between participants indicates they all had different experiences with the same BCI- AAC system. Therefore, these varying perceptions continue to highlight their importance of considering the user experience in BCI-AAC development, along with the creation of feature matching procedures to help 
Table 3. Summary statistics.

\begin{tabular}{|c|c|c|c|c|c|}
\hline Participant/area & Mean & $\begin{array}{l}\text { Standard } \\
\text { Deviation }\end{array}$ & Range & $\begin{array}{l}\text { Significance: } \\
\text { Performance }\end{array}$ & $\begin{array}{l}\text { Significance: } \\
\text { Satisfaction }\end{array}$ \\
\hline A1: Fatigue-Pre & 3.75 & 1.66 & $1-6$ & $p=.212$ & \\
\hline A2: Fatigue-Pre & 2.67 & 1.56 & $1-5$ & $p=.102$ & \\
\hline A3: Fatigue-Pre & 3.88 & 1.63 & $1-7.5$ & $p=.365$ & \\
\hline A4: Fatigue-Pre & 5.49 & 1.38 & $2-7$ & $p=.208$ & \\
\hline A1: Fatigue: Pre-Post & -1.08 & 1.31 & $-4-1$ & & $p=.221$ \\
\hline A2: Fatigue: Pre-Post & 0.417 & 1.31 & $-2-2$ & & $p=.586$ \\
\hline A3: Fatigue: Pre-Post & 1.29 & 2.11 & $-1-6$ & & $p=.207$ \\
\hline A4: Fatigue: Pre-Post & 0.75 & 1.22 & $-1-2$ & & $p=.184$ \\
\hline A1: Satisfaction & 7.33 & .985 & $5-9$ & ${ }^{*} p<.05$ & \\
\hline A2: Satisfaction & 6.75 & 1.82 & $4-9$ & $p=.245$ & \\
\hline A3: Satisfaction & 5.08 & 1.73 & $2-7$ & ${ }^{*} p<.05$ & \\
\hline A4: Satisfaction & 7.25 & .866 & $6-9$ & $p=.674$ & \\
\hline A1: Frustration & 3.25 & 1.36 & $1-5$ & $p=.877$ & $p=.736$ \\
\hline A2: Frustration & 5.25 & 1.76 & $2-8$ & $p=.452$ & ${ }^{*} p<.05$ \\
\hline A3: Frustration & 6.08 & 1.16 & $4-8$ & $p=.463$ & $p=.142$ \\
\hline A4: Frustration & 4.17 & 1.85 & $2-7$ & $p=.097$ & ${ }^{*} p<.05$ \\
\hline A1: Mental Effort & 6.17 & 1.34 & $3-8$ & $p=.115$ & $p=.252$ \\
\hline A2: Mental Effort & 6.92 & 1.16 & $4-8$ & $p=.116$ & $p=.7$ \\
\hline A3: Mental Effort & 7 & 1.35 & $5-9$ & $p=.394$ & $p=.459$ \\
\hline A4: Mental Effort & 5.17 & 1.85 & $1-7$ & $p=.181$ & $p=.371$ \\
\hline A1: Physical Effort & 1.42 & 0.669 & $1-3$ & $p=.795$ & $p=.153$ \\
\hline A2: Physical Effort & 4.25 & 1.42 & $2-6$ & $p=.344$ & $p=.284$ \\
\hline A3: Physical Effort & 5.08 & 1.68 & $1-7$ & $p=.734$ & $p=.877$ \\
\hline A4: Physical Effort & 2.25 & .622 & $1-3$ & $p=.621$ & $p=.068$ \\
\hline A1: Overall Hardness & 4.58 & 1.73 & $2-8$ & $p=.974$ & $p=.703$ \\
\hline A2: Overall Hardness & 6.17 & 1.34 & $4-7$ & $p=.530$ & $p=.469$ \\
\hline A3: Overall Hardness & 6.5 & 1.24 & $4-9$ & $p=.658$ & $p=.7$ \\
\hline A4: Overall Hardness & 3.42 & 1.31 & $2-6$ & $p=.751$ & $p=.392$ \\
\hline
\end{tabular}

Summary statistics for number scale measures in relation to $\mathrm{BCl}-\mathrm{AAC}$ performance and satisfaction. Items of statistical significance marked with a * in bold.

ensure individuals are matched to a device that is most likely to support communication success. All participants gave their highest ratings for BCI-AAC use in the area of mental effort with average scores bordering and reaching fairly high for participants A2 (6.92), and A3 (7). While it is unclear whether ratings of mental effort would have decreased for these participants with improved levels of BCI-AAC control, in a feature matching context, these high levels of mental effort may indicate that testing with a different BCI-AAC devices may be warranted to explore the impacts of other BCI-AAC systems on their experience and device preferences. In comparison, the lowest average 
score for mental effort was indicated by A4 (5.17; neutral) who had the largest slope associated with his BCI-AAC learning trajectory. In addition, while non-significant, our study findings demonstrated a negative trend between mental effort ratings and BCI-AAC performance $\left(r_{s}=-.478\right.$ to -.479$)$ for participants A1 and A2. Associations between decreased mental effort and improving levels of BCI-AAC performance have been reported previously (e.g., Witte et al., 2013). Therefore, taken together, further research exploring the factors contributing to increased ratings of mental effort, and strategies to reduce mental effort (e.g., helping individuals to not overthink BCI-AAC motor learning) may help to lower individuals' perceived effort with use their BCI-AAC system and improve performance, which may also ultimately contribute toward improving BCI-AAC acceptance.

Fatigue is also an important consideration during BCI-AAC assessment and training that may impact attention (Boksem et al., 2005) and vigilance (Oken et al., 2018), possibly decreasing EEG signal changes (and thus signal-to-noise ratios) needed to detect event-related desynchronization (Kasahara et al., 2012) and thus decreasing motorbased BCI-AAC performance. However, our study revealed that how we incorporate fatigue ratings into tools evaluating the individuals BCI-AAC experience may be complex and individualized, requiring further consideration for its appropriate use. Based on previous reports that BCI-AAC control requires high levels of effort (e.g., Chavarriaga et al., 2017), we expected that pre- to post-fatigue ratings would be high for the present BCI-AAC control paradigm. However, surprisingly, while average ratings of mental effort across participants ranged from neutral (5) to fairly high (7), average pre- to post-fatigue ratings were very low, ranging from -1 to 1 . Negative pre- to post-fatigue ratings indicate a perception of less fatigue following BCI-AAC use, possibly due to participation in an engaging activity, which was corroborated by participant A3 in particular. Furthermore, differences between mental effort and pre- to post-fatigue ratings may be due to participants' definition of the term fatigue, which may possibly be influenced by neuromotor impairment severity. More specifically, three participants indicated the term fatigue better reflects physical effort, which was generally low for this BCI-AAC system. However, A3, indicated that she uses the term in a manner generally synonymous with mental effort, possibly due to her greater impairment severity and 
limited physical movement. Therefore, it is important that future BCIAAC training sessions provide sufficient options for rating effort (e.g., mental effort, physical effort) and consider how each participant defines fatigue to allow for individualized and precise ratings of person-centered factors associated with BCI-AAC use. For instance, if fatigue is reported high and was associated with relatively low BCI-AAC performance, it is not possible to assume that reducing mental effort needed to control the BCI-AAC will improve performance. In fact, for individuals who relate fatigue to physical rather than mental effort, some other BCI-AAC adaptation may be needed to improve BCI-AAC performance and acceptance.

\section{Factors that impact satisfaction with the BCI-AAC system}

The results of our study suggest factors that influence satisfaction with the present motor-based BCI-AAC system are mixed. Overall, participants A1 and A4 who achieved higher levels of BCI-AAC performance in comparison to $\mathrm{A} 2$ and $\mathrm{A} 3$ provided higher mean ratings for satisfaction ( $\mathrm{A} 1=7.33, \mathrm{~A}_{4}=7.25, \mathrm{~A} 2=6.75, \mathrm{~A} 3=5.08$ ). However, looking in further detail, satisfaction ratings were positively correlated with performance for participants A1 and A3 but were primarily driven by levels of frustration for participants A2 and A4. Satisfaction for participant A4 may also have been related to physical effort, with correlations approaching significance $(p=.068)$. Individual adaptions in factors such as rate and signal processing were not implemented to allow for fair comparisons across participants. Therefore, overall satisfaction scores may have been increased through personalization. Alongside Lorenz et al. (2014), these findings continue to support that evaluations of the user experience should include a holistic approach considering factors related to both usability (i.e., pragmatic factors) and appeal (i.e. hedonic factors). Therefore, based on study findings, future BCI-AAC research and intervention paradigms are encouraged to include outcome measures such as frustration and physical effort ratings, to help provide person-centered context on participant satisfaction with BCI-AAC technology and how that reflects on traditional performance measures.

Through understanding factors behind BCI-AAC satisfaction and acceptance, BCI-AAC research may seek to optimize training strategies, 
bring BCI-AAC research further in line with existing clinical practices helping to decrease abandonment issues, and inform future research directions in BCI-AAC development. For instance, studies that evaluate individuals' experiences with BCI-AAC use, both initially and over time, may help prepare clinicians to set realistic expectations during BCI-AAC training. Through setting realistic expectations for BCIAAC performance and the associated levels of effort, satisfaction, and fatigue, the AAC team may help lower levels of frustration associated with BCI-AAC, helping increase device acceptance (Johnson et al., 2006; Moorcroft et al., 2019). Furthermore, BCI-AAC performance can be highly variable both between and within participants (Ahn \& Jun, 2015; Pitt \& Brumberg, 2021), and it is plausible that inconsistent performance outcomes may impact frustration levels. Therefore, in addition to identifying how person-centered strengths impact BCI-AAC outcomes, user-centered BCI-AAC development, and feature matching procedures may be supported by identifying how person-centered characteristics are associated with performance variability. Understanding variability may further help clinicians set realistic expectations regarding BCI-AAC learning while also helping inform BCI-AAC development by identifying factors beyond the control of engineering solution.

\section{Limitations and future directions}

The results of our study highlighted multiple factors for consideration in understanding individuals' perceptions of motor-based BCIAAC during training. However, further research is needed with larger sample sizes to corroborate these findings and develop clinically based standardized guidelines for obtaining feedback from individuals during BCI-AAC training. In addition, further work is needed to elucidate a full range of user-centered factors that are in supporting BCI-AAC success and generalize these findings to 1) other types of BCI-AAC systems (e.g., P300, steady state visual evoked potential), and 2) other adult populations with severe physical impairments (e.g., locked in syndrome, spinal cord injury), in addition to children with severe physical impairments, who may have different communication wants and needs in comparison to adult populations. Individuals participating in 
this study did not utilize AAC as their primary communication method, with only A3 using an eye-gaze-based system for social media and communication support. Therefore, how individuals' experiences differed between use of the BCI-AAC system, and their current device are unclear, and require further exploration. Further, the user experience was based on a 20-minute period of real-time BCI-AAC use per session. Therefore, how discussed factors change during extended BCIAAC control tasks may also help elucidate more real-life user experiences with BCI-AAC control.

Acknowledgments The authors would like to thank Chavis Lickvar-Armstrong for her assistance with this project.

Funding This work was supported in part by the Texas Woman's University Woodcock Institute Research Grant, the ASHA Foundation New Century Scholars Grant, the University of Kansas Summer Research Scholarship, and the National Institute on Deafness and other Communication Disorders (NIDCD R01-DC016343).

\section{References}

Ahn, M., \& Jun, S. C. (2015). Performance variation in motor imagery braincomputer interface: A brief review. Journal of Neuroscience Methods, 243, 103110. https://doi.org/10.1016/j.jneumeth.2015.01.033

Andresen, E. M., Fried-Oken, M., Peters, B., \& Patrick, D. L. (2016). Initial constructs for patient-centered outcome measures to evaluate brain-computer interfaces. Disability and Rehabilitation: Assistive Technology, 11(7), 548-557. https://doi.org/10.3109/17483107.2015.1027298

Beukelman, D., \& Light, J. (2020). Augmentative and alternative communication: Supporting children and adults with complex communication needs (5th ed.). Paul H. Brookes Publishing Co.

Bircanin, F., Ploderer, B., Sitbon, L., Bayor, A. A., \& Brereton, M. (2019, December). Challenges and opportunities in using augmentative and alternative communication (AAC) technologies: Design considerations for adults with severe disabilities. In Proceedings of the 31st Australian Conference on Human-Computer-Interaction (pp. 184-196). New York, NY, USA: Association for Computing Machinery.

Blain-Moraes, S., Schaff, R., Gruis, K. L., Huggins, J. E., \& Wren, P. A. (2012). Barriers to and mediators of brain-computer interface user acceptance: Focus group findings. Ergonomics, 55(5), 516-525. https://doi.org/10.1080/00140139 .2012 .661082 
Boksem, M. A., Meijman, T. F., \& Lorist, M. M. (2005). Effects of mental fatigue on attention: An ERP study. Cognitive Brain Research, 25(1), 107-116. https://doi. org/10.1016/j.cogbrainres.2005.04.011

Brumberg, J. S., Pitt, K. M., Mantie-Kozlowski, A., \& Burnison, J. D. (2018). Braincomputer interfaces for augmentative and alternative communication: A tutorial. American Journal of Speech-Language Pathology, 27(1), 1-12. https:// doi.org/10.1044/2017_AJSLP-16-0244

Cedarbaum, J. M., \& Stambler, N. (1997). Performance of the amyotrophic lateral sclerosis functional rating scale (ALSFRS) in multicenter clinical trials. Journal of the Neurological Sciences, 152(Suppl. 1), s1-s9. https://doi.org/10.1016/ So022-510X(97)00237-2

Chavarriaga, R., Fried-Oken, M., Kleih, S., Lotte, F., \& Scherer, R. (2017). Heading for new shores! Overcoming pitfalls in BCI design. Brain-Computer Interfaces, 4(1-2), 6o-73. https://doi.org/10.1080/2326263X. 2016.1263916

Combaz, A., Chatelle, C., Robben, A., Vanhoof, G., Goeleven, A., Thijs, V., . . . Laureys, S. (2013). A comparison of two spelling brain-computer interfaces based on visual P3 and SSVEP in locked-in syndrome. PLoS One, 8(9), e73691. https://doi.org/10.1371/journal.pone.0073691

Fager, S., Fried-Oken, M., Jakobs, T., \& Beukelman, D. R. (2019). New and emerging access technologies for adults with complex communication needs and severe motor impairments: State of the science. Augmentative and Alternative Communication, 35(1), 13-25. https://doi.org/10.1080/07434618.2 018.1556730

Friedrich, E. V., Scherer, R., \& Neuper, C. (2013). Long-term evaluation of a 4-class imagery-based brain-computer interface. Clinical Neurophysiology, 124(5), 916-927. https://doi.org/10.1016/j.clinph. 2012.11.010

Geronimo, A., Stephens, H. E., Schiff, S. J., \& Simmons, Z. (2015). Acceptance of brain-computer interfaces in amyotrophic lateral sclerosis. Amyotrophic Lateral Sclerosis and Frontotemporal Degeneration, 16(3-4), 258-264. https:// doi.org/10.3109/21678421.2014.969275

Huggins, J. E., Wren, P. A., \& Gruis, K. L. (2011). What would brain-computer interface users want? Opinions and priorities of potential users with amyotrophic lateral sclerosis. Amyotrophic Lateral Sclerosis, 12 (5), 318-324. https://doi.org/10.3109/17482968.2011.572978

Johnson, J. M., Inglebret, E., Jones, C., \& Ray, J. (2006). Perspectives of speech language pathologists regarding success versus abandonment of AAC. Augmentative and Alternative Communication, 22(2), 85-99. https://doi. org/10.1080/07434610500483588

Kasahara, T., Terasaki, K., Ogawa, Y., Ushiba, J., Aramaki, H., \& Masakado, Y. (2012). The correlation between motor impairments and event-related desynchronization during motor imagery in ALS patients. BMC Neuroscience, 13(1), 66. https://doi.org/10.1186/1471-2202-13-66

Kögel, J., Schmid, J. R., Jox, R. J., \& Friedrich, O. (2019). Using brain-computer interfaces: A scoping review of studies employing social research methods. BMC Medical Ethics, 2o(1), 1-17. https://doi.org/10.1186/s12910-019-0354-1 
Kübler, A., Holz, E. M., Riccio, A., Zickler, C., Kaufmann, T., Kleih, S. C., Mattia, D., Hoogerwerf, E.-J., Mattia, D., \& Staiger-Sälzer, P. (2014). The user-centered design as novel perspective for evaluating the usability of BCI-controlled applications. PLoS One, 9(12), e112392. https://doi.org/10.1371/journal. pone.0112392

Kuhlman, W. N. (1978). Functional topography of the human mu rhythm. Electroencephalography and Clinical Neurophysiology, 44(1), 83-93. https:// doi.org/10.1016/0013-4694(78)90107-4

Lorenz, R., Pascual, J., Blankertz, B., \& Vidaurre, C. (2014). Towards a holistic assessment of the user experience with hybrid BCIs. Journal of Neural Engineering, 11(3), 035007. https://doi.org/10.1088/1741-2560/11/3/035007

Lotte, F., \& Guan, C. (2010). Regularizing common spatial patterns to improve BCI-AAC designs: Unified theory and new algorithms. IEEE Transactions on Biomedical Engineering, 58(2), 355-362. https://doi.org/10.1109/ TBME.2010.2082539

Mak, J. N., \& Wolpaw, J. R. (2009). Clinical applications of brain-computer interfaces: Current state and future prospects. IEEE Reviews in Biomedical Engineering, 2, 187-199. https://doi.org/10.1109/RBME.2009.2035356

McHugh, M. L. (2012). Interrater reliability: The kappa statistic. Biochemia Medica: Biochemia Medica, 22(3), 276-282. https://doi.org/10.11613/ BM.2012.031

Moorcroft, A., Scarinci, N., \& Meyer, C. (2019). A systematic review of the barriers and facilitators to the provision and use of low-tech and unaided AAC systems for people with complex communication needs and their families. Disability and Rehabilitation: Assistive Technology, 14(7), 710-731. https://doi.org/10.108 o/17483107.2018.1499135

Nijboer, F., Birbaumer, N., \& Kübler, A. (2010). The influence of psychological state and motivation on brain-computer interface performance in patients with amyotrophic lateral sclerosis - a longitudinal study. Frontiers in Neuroscience, 4(55), 1-13. https://doi.org/10.3389/fnins. 2010.00055

Oken, B., Memmott, T., Eddy, B., Wiedrick, J., \& Fried-Oken, M. (2018). Vigilance state fluctuations and performance using brain-computer interface for communication. Brain-Computer Interfaces, 5(4), 146-156. https://doi.org/10.1 080/2326263X.2019.1571356

Oostenveld, R., \& Praamstra, P. (2001). The five percent electrode system for highresolution EEG and ERP measurements. Clinical Neurophysiology, 112(4), 713719. https://doi.org/10.1016/S1388-2457(00)00527-7

Peters, B., Mooney, A., Oken, B., \& Fried-Oken, M. (2016). Soliciting BCI user experience feedback from people with severe speech and physical impairments. Brain-Computer Interfaces, 3(1), 47-58. https://doi.org/10.1080/23262 63X.2015.1138056

Pfurtscheller, G., \& Da Silva, F. L. (1999). Event-related EEG/MEG synchronization and desynchronization: Basic principles. Clinical Neurophysiology, 110(11), 1842-1857. https://doi.org/10.1016/S1388-2457(99)o0141-8 
Pfurtscheller, G., \& Neuper, C. (2009). Dynamics of sensorimotor oscillations in a motor task. In Graimann, B., Pfurtscheller, G., \& Allison, B. (Eds.), Braincomputer interfaces (pp. 47-64). Springer.

Pitt, K., \& Brumberg, J. (2020). A screening protocol incorporating braincomputer interface feature matching considerations for augmentative and alternative communication. Assistive Technology, 32(3), 161- 172. https://doi. org/10.1080/10400435.2018.1512175

Pitt, K., \& Brumberg, J. (2021). Evaluating person-centered factors associated with brain-computer interface access to commercial augmentative and alternative communication paradigm. Assistive Technology, 1-10. https://doi.org/10.1080/ $\underline{10400435.2021 .1872737}$

Pitt, K. M., \& Brumberg, J. S. (2018a). Guidelines for feature matching assessment of brain-computer interfaces for augmentative and alternative communication. American Journal of Speech-Language Pathology, 27 (3), 950-964. https://doi. org/10.1044/2018 AJSLP-17-0135

Pitt, K. M., Brumberg, J. S., \& Pitt, A. R. (2019). Considering augmentative and alternative communication research for brain-computer interface practice. Assistive Technology Outcomes and Benefits, 13 (Summer), 1-20. www.atia. org/atob

Vaughan, T. M., McFarland, D. J., Schalk, G., Sarnacki, W. A., Krusienski, D. J., Sellers, E. W., \& Wolpaw, J. R. (2006). The Wadsworth BCI research and development program: At home with BCI. IEEE Transactions on Neural Systems and Rehabilitation Engineering, 14(2), 229-233. https://doi.org/10.1109/ TNSRE.2006.875577

Witte, M., Kober, S. E., Ninaus, M., Neuper, C., \& Wood, G. (2013). Control beliefs can predict the ability to up-regulate sensorimotor rhythm during neurofeedback training. Frontiers in Human Neuroscience, 7, 478. https://doi. org/10.3389/fnhum.2013.00478

Woolley, S. C. (2014). ALS cognitive behavioral screen manual. The Forbes Norris MDA/ALS Research Center.

Woolley, S. C., York, M. K., Moore, D. H., Strutt, A. M., Murphy, J., Schulz, P. E., \& Katz, J. S. (2010). Detecting frontotemporal dysfunction in ALS: Utility of the ALS cognitive behavioral screen (ALS-CBS). Amyotrophic Lateral Sclerosis, 11(3), 303-311. https://doi.org/10.3109/17482961003727954 
Supplementary Material A. Number scale ratings of participant perspectives.

\section{Fatigue}

1a. Please rate your current level of fatigue (prior to BCI-AAC use) using the scales below

\begin{tabular}{ccccccccc} 
Normal & & Mild & & Moderate & & High & \multicolumn{2}{c}{ Extremely fatigued } \\
$\mathbf{1}$ & $\mathbf{2}$ & $\mathbf{3}$ & $\mathbf{4}$ & $\mathbf{5}$ & $\mathbf{6}$ & $\mathbf{7}$ & $\mathbf{8}$ & $\mathbf{9}$
\end{tabular}

1b. Following today's free spelling tasks, indicate your level of fatigue on a scale of $1-9,9$ being extremely_fatigued, to 1 being normal

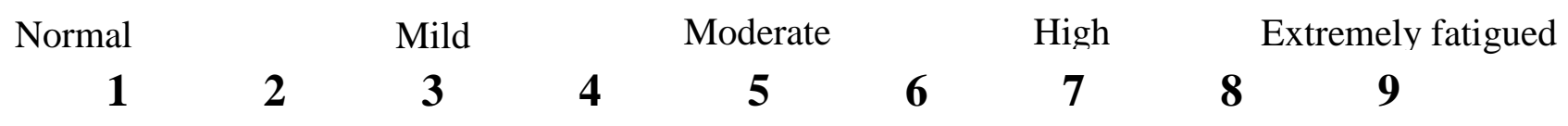

1c. Post minus pre free spelling ratings of fatigue:

1d. How would you define fatigue?

\section{Device Satisfaction}

1. Following today's spelling tasks, how satisfied are you with this BCI-AAC system?

\begin{tabular}{ccccccccc} 
Very unsatisfied & \multicolumn{3}{c}{ Mildly unsatisfied } & Neutral & \multicolumn{2}{c}{ Mildly satisfied } & & Very satisfied \\
$\mathbf{1}$ & $\mathbf{2}$ & $\mathbf{3}$ & $\mathbf{4}$ & $\mathbf{5}$ & $\mathbf{6}$ & $\mathbf{7}$ & $\mathbf{8}$ & $\mathbf{9}$
\end{tabular}

2. During today's spelling tasks, what level of frustration did you experience with using the $\mathrm{BCI}$ ?

$\begin{array}{ccccccccc}\text { Very low } & & \text { Fairly low } & & \text { Neutral } & & \text { Fairly high } & & \text { Very high } \\ \mathbf{1} & \mathbf{2} & \mathbf{3} & \mathbf{4} & \mathbf{5} & \mathbf{6} & \mathbf{7} & \mathbf{8} & \mathbf{9}\end{array}$


Evaluating the Perspectives of Those with SPI

3. During today's spelling tasks, how much physical effort was required to operate the BCI?

$\begin{array}{ccccccccc}\text { Very low } & & \text { Fairly low } & & \text { Neutral } & & \text { Fairly high } & & \text { Very high } \\ \mathbf{1} & \mathbf{2} & \mathbf{3} & \mathbf{4} & \mathbf{5} & \mathbf{6} & \mathbf{7} & \mathbf{8} & \mathbf{9}\end{array}$

4. During today's spelling tasks, how much mental effort or concentration was required to operate the BCI?

$\begin{array}{ccccccccc}\text { Very low } & & \text { Fairly low } & & \text { Neutral } & & \text { Fairly high } & & \text { Very high } \\ \mathbf{1} & \mathbf{2} & \mathbf{3} & \mathbf{4} & \mathbf{5} & \mathbf{6} & \mathbf{7} & \mathbf{8} & \mathbf{9}\end{array}$

5. Overall how hard did you have to work to complete todays spelling tasks?

\begin{tabular}{|c|c|c|c|c|c|c|c|}
\hline Very easy & & Fairly easy & & Neutral & & Fairly hard & \\
\hline 1 & 2 & 3 & 4 & 5 & 6 & 7 & 8 \\
\hline
\end{tabular}


Supplemental Material B. Recurring number scale ratings for each participant and evaluation area across the 12 BCI-AAC training sessions (S1-S12).

\begin{tabular}{lllllllllllll}
\hline Participant/area & S1 & S2 & S3 & S4 & S5 & S6 & S7 & S8 & S9 & S10 & S11 & S12
\end{tabular}

\begin{tabular}{lllllllllllllll}
\hline A1: Fatigue- & 6 & 3 & 6 & 5 & 5 & 4 & 1 & 4 & 3 & 4 & 3 & 1
\end{tabular}

Pre

$\begin{array}{lllllllllllllll}\text { A1: Fatigue- } & 2 & 2 & 5 & 3 & 5 & 3 & 1 & 3 & 1 & 3 & 2 & 2\end{array}$

Post

A1: Fatiguewithin

$$
\begin{array}{llllllllllll}
-4 & -1 & -1 & -2 & 0 & -1 & 0 & -1 & -2 & -1 & -1 & 1
\end{array}
$$

$\begin{array}{lllllllllllll}\text { A1: Frustration } & 2 & 2 & 4 & 5 & 4 & 5 & 2 & 5 & 3 & 3 & 1 & 3\end{array}$ A1:

Satisfaction

$\begin{array}{llllllllllll}5 & 9 & 7 & 7 & 7 & 8 & 8 & 7 & 8 & 7 & 7 & 8\end{array}$

A1: Mental

Effort

$\begin{array}{llllllllllll}7 & 7 & 8 & 6 & 7 & 3 & 5 & 6 & 7 & 7 & 6 & 5\end{array}$
A1: Physical
Effort

$\begin{array}{llllllllllll}1 & 1 & 2 & 3 & 1 & 1 & 1 & 1 & 1 & 2 & 2 & 1\end{array}$

A1: Overall

Hardness

$\begin{array}{llllllllllll}8 & 4 & 3 & 5 & 2 & 6 & 5 & 5 & 5 & 2 & 4 & 6\end{array}$

A2: Fatigue-
Pre

A2: Fatigue-

Post 
Evaluating the Perspectives of Those with SPI

A2: Fatigue-

within

$\begin{array}{llllllllllll}2 & 1 & 0 & 0 & 2 & 2 & 1 & -2 & -1 & -1 & 1 & 0\end{array}$ $\begin{array}{lllllllllllll}\text { A2: Frustration } & 6 & 6 & 5 & 5 & 6 & 3 & 3 & 2 & 7 & 6 & 6 & 8\end{array}$ A2:

Satisfaction

$\begin{array}{llllllllllll}8 & 7 & 7 & 9 & 7 & 8 & 8 & 9 & 4 & 5 & 5 & 4\end{array}$

A2: Mental

Effort

$\begin{array}{llllllllllll}8 & 8 & 7 & 7 & 7 & 8 & 6 & 4 & 7 & 6 & 8 & 7\end{array}$

A2: Physical

Effort

$\begin{array}{llllllllllll}5 & 5 & 2 & 3 & 5 & 6 & 3 & 3 & 6 & 3 & 6 & 4\end{array}$

A2: Overall

Hardness

$\begin{array}{llllllllllll}7 & 7 & 6 & 7 & 7 & 7 & 4 & 4 & 7 & 4 & 7 & 7\end{array}$

A3: Fatigue-

Pre

$\begin{array}{llllllllllll}4 & 3 & 5 & 4 & 2 & 1 & 4 & 7.5 & 4 & 5 & 3 & 4\end{array}$

A3: Fatigue-

Post

$\begin{array}{llllllllllll}6 & 4 & 4 & 5 & 7 & 7 & 4 & 8 & 4 & 5 & 4 & 4\end{array}$

A3: Fatigue-

within

$\begin{array}{llllllllllll}2 & 1 & -1 & 1 & 5 & 6 & 0 & 5 & 0 & 0 & 1 & 0\end{array}$

$\begin{array}{lllllllllllll}\text { A3: Frustration } & 6 & 6 & 7 & 4 & 7 & 8 & 5 & 6 & 7 & 5 & 7 & 5\end{array}$

A3:

Satisfaction

$\begin{array}{llllllllllll}7 & 3 & 6 & 5 & 6 & 2 & 7 & 3 & 5 & 6 & 4 & 7\end{array}$

A3: Mental

Effort

$\begin{array}{llllllllllll}8 & 9 & 9 & 8 & 8 & 6 & 6 & 5 & 6 & 6 & 6 & 7\end{array}$ 
Evaluating the Perspectives of Those with SPI
A3: Physical
Effort

$\begin{array}{llllllllllll}1 & 3 & 6 & 7 & 6 & 6 & 5 & 5 & 5 & 5 & 5 & 7\end{array}$

A3: Overall

Hardness

$\begin{array}{llllllllllll}6 & 9 & 7 & 7 & 8 & 6 & 6 & 4 & 6 & 6 & 6 & 7\end{array}$

A4: Fatigue-

Pre

$\begin{array}{llllllllllll}4 & 3 & 5 & 4 & 2 & 5 & 7 & 5 & 4 & 4 & 6 & 6\end{array}$

A4: Fatigue-

Post

$\begin{array}{llllllllllll}3 & 3 & 7 & 5 & 4 & 6 & 8 & 7 & 6 & 3 & 5 & 7\end{array}$

A4: Fatigue-

within

$\begin{array}{lllllllllllll}-1 & 0 & 2 & 1 & 2 & 1 & 1 & 2 & 2 & -1 & -1 & 1\end{array}$

$\begin{array}{lllllllllllll}\text { A4: Frustration } & 7 & 5 & 6 & 3 & 5 & 2 & 2 & 6 & 6 & 2 & 3 & 3\end{array}$

A4:

Satisfaction

$\begin{array}{lllllllllllll}7 & 7 & 6 & 8 & 7 & 9 & 7 & 7 & 6 & 8 & 7 & 8\end{array}$

A4: Mental

Effort

$\begin{array}{llllllllllll}6 & 4 & 4 & 7 & 4 & 6 & 1 & 4 & 7 & 7 & 7 & 5\end{array}$

A4: Physical

Effort

$\begin{array}{llllllllllll}2 & 2 & 3 & 2 & 2 & 1 & 2 & 3 & 3 & 3 & 2 & 2\end{array}$

A4: Overall

Hardness

$\begin{array}{llllllllllll}3 & 2 & 5 & 4 & 2 & 3 & 2 & 5 & 6 & 3 & 3 & 3\end{array}$ 